\title{
Impact of therapy on quality of life, neurocognitive function and their correlates in glioblastoma multiforme: a review
}

\author{
Roger Henriksson • Thomas Asklund • \\ Hans Skovgaard Poulsen
}

Received: 19 December 2010/Accepted: 3 March 2011/Published online: 6 April 2011

(c) The Author(s) 2011. This article is published with open access at Springerlink.com

\begin{abstract}
The maintenance of quality of life $(\mathrm{QoL})$ in patients with high-grade glioma is an important endpoint during treatment, particularly in those with glioblastoma multiforme (GBM) given its dismal prognosis despite limited advances in standard therapy. It has proven difficult to identify new therapies that extend survival in patients with recurrent GBM, so one of the primary aims of new therapies is to reduce morbidity, restore or preserve neurologic functions, and the capacity to perform daily activities. Apart from temozolomide, cytotoxic chemotherapeutic agents do not appear to significantly impact response or survival, but produce toxicity that is likely to negatively impact QoL. New biological agents, such as bevacizumab, can induce a clinically meaningful proportion of durable responses among patients with recurrent GBM with an acceptable safety profile. Emerging evidence suggests that bevacizumab produces an improvement or preservation of neurocognitive function in GBM patients, suggestive of QoL improvement, in most poorprognosis patients who would otherwise be expected to show a sudden and rapid deterioration in QoL.
\end{abstract}

R. Henriksson · T. Asklund

Department of Radiation Sciences and Oncology,

Umeå University Hospital, Umea, Sweden

R. Henriksson · H. S. Poulsen

Radiumhemmet, Karolinska Hospital, Stockholm, Sweden

H. S. Poulsen

Department of Oncology, Finsencenter, University Hospital,

9 Blegdamsvej, 2100 Copenhagen, Denmark

H. S. Poulsen $(\square)$

Department of Radiation Biology, Section 6321,

Finsencenter, University Hospital, 9 Blegdamsvej,

2100 Copenhagen, Denmark

e-mail: Hans.Skovgaard.Poulsen@rh.regionh.dk
Keywords Quality of life - Glioblastoma multiforme · Bevacizumab

\section{Introduction}

High-grade gliomas, WHO grade III or IV [1, 2], are the most common primary brain tumors in adults $[1,3]$. WHO grade IV tumors are almost exclusively (80-90\%) glioblastoma multiforme (GBM), which are the most common high-grade glioma (40-45\%) [1,3], the most common form of brain tumor overall $(12-15 \%)[1,3]$, and the most aggressive malignant primary brain tumor [4]. Despite limited significant advances in standard therapy, notably temozolomide, median overall survival (OS) remains low: 15 months for newly-diagnosed GBM [5, 6] and 5-7 months for recurrent/relapsed GBM [7-10]. In a clinical trial setting, the current standard of care (radiotherapy plus temozolomide followed by 6 cycles of adjuvant temozolomide) provided 2- and 5-year survival rates of 27 and 10\% for patients with newly diagnosed GBM [6]. Thus, an unmet medical need for improved therapeutic options remains.

Given the poor prognosis of GBM, the primary objectives of therapy are to reduce morbidity, restore or preserve neurologic functions and the capacity to perform daily activities as long as possible [11]. The aim of this review is to examine the impact of GBM therapy on QoL, neurocognitive function, and their correlates.

\section{Neurocognitive functioning and QoL}

It is well recognized that impairment of neurocognitive functioning, resulting in behavioral, emotional, and 
intellectual difficulties, occurs in nearly all patients with brain tumors and eventually compromises their independence [12]. This impairment is related to a combination of various factors, including the tumor itself, tumor-related epilepsy, treatment, and patient-related factors (e.g., age, psychological distress) [13-16]. However, there have been relatively few well-powered longitudinal studies of neurocognitive function in patients with high-grade glioma [17]. Neurocognitive function is an important determinant of QoL [18-20]. Not surprisingly, neurocognitive function assessments have been incorporated as major components of patient assessments, along with common and widely used questionnaires to assess health-related QoL (HRQoL), e.g., European Organization for Research and Treatment of Cancer (EORTC) Core Quality of Life Questionnaire (QLQ-C30), and Functional Assessment of Cancer Therapy (FACT) cancer-specific scales [21]. Indeed, neurocognitive function has been shown to be a valid predictor of long-term QoL [22-24].

As life expectancy in high-grade glioma, and particularly in recurrent GBM, is so short, issues relating to $\mathrm{QoL}$ are immensely important to patients and their caregivers [17]. This is especially important in relation to new treatments in recurrent GBM that do not yet have evidence supporting their contribution to extended survival but may significantly delay the expected steep QoL deterioration occurring after progression following standard therapies [17]. Unfortunately, QoL data are difficult to collect in cancer patients because they may be unwilling to complete the questionnaire when they are feeling unwell. Furthermore, repeated application of lengthy, formal HR-QoL questionnaires can represent a major and impractical burden for patients [25]. Also, the analysis of QoL data is challenging due to the high rates of non-random missing QoL values that may be linked to patients' QoL status, and if ignored may introduce bias in the interpretation of results [26]. Interpretation of the impact of standard and new therapies on QoL in GBM patients is consequently problematic, even when attempting to classify their effect into the three broad categories of negative, positive, or neutral.

To overcome these challenges, changes in neurocognitive functioning may be taken as a proxy for QoL changes. Assessment of neurocognitive function can therefore represent a practical surrogate for formal QoL assessment of patients with recurrent GBM. In addition, besides grade and age, performance status in patients with newly diagnosed glioma is an independent prognostic marker. Thus, it is plausible to assume that neurocognitive function, irrespective of clinical stage, may also have prognostic implications even after initiation of therapy and during the course of treatment.

\section{Treatment impact on QoL and its correlates}

HR-QoL in patients with high-grade glioma has recently been reviewed in detail [17], which noted problems associated with interpretation of different studies and the paucity of robust HR-QoL information derived from wellpowered randomized controlled trials. Among the seven randomized controlled trials of new treatments published from 2002 to 2007 they identified for high-grade glioma, there was no or little difference between treatment groups at baseline or follow-up evaluation. These authors suggested that standard multidimensional HR-QoL questionnaires might therefore contain too many items and consequently lack sensitivity to detect QoL changes in patients with high-grade glioma. Simpler, practical, and more sensitive instruments (such as cognitive function) are therefore needed to study QoL changes in relation to therapy in high-grade glioma, and, thus, the confounding factor of missing substantial follow-up data (primarily related to dropouts) needs to be addressed.

\section{Standard therapy}

Neurosurgery and/or radiotherapy are still fundamental elements of standard therapy for patients with high-grade glioma. It is well recognized that surgery may initially, at least temporarily, improve QoL dramatically in a significant proportion of patients with severe symptoms related to increased intracranial pressure [3]. Conversely, radiotherapy may decrease QoL in some patients from adverse effects such as hair loss, fatigue, somnolence, or cognitive problems [26]. The influence of radiotherapy on neurocognitive function has recently been reviewed [27]. It is clear that tumor recurrence and short-term survival are confounding variables, but it is generally agreed that shortterm memory and progression of dementia are observed in many patients treated with brain irradiation. The impact of adjunctive therapy with corticosteroids and antiepileptic medication has also been extensively studied. While the presence and severity of epileptic seizures and/or the use of antiepileptic medication have been significantly associated with cognitive deficits in patients with low-grade gliomas, more so than the effects of radiotherapy [28-30], the effects of antiepileptic medication on neurocognitive functioning and QoL have been less extensively studied in patients with high-grade glioma, although some studies have reported a negative impact [31].

The negative effects of corticosteroid use on neurocognitive function and/or QoL are well documented in healthy subjects $[32,33]$ and in various disease states, such as leukemia [34]. While it appears accepted that the use 
of high-dose corticosteroids has a clinically significant negative impact on neurocognitive function in glioma, there would appear to be no published information in this indication. However, it is documented that corticosteroid use in primary brain tumors and/or metastases caused adverse effects [35, 36] which would be expected to decrease QoL.

Assessment of QoL is important with cytotoxic chemotherapy, particularly when survival benefit may be marginal and has to be balanced against any negative contribution of significant toxicity. Among newly diagnosed GBM patients randomized to radiotherapy alone or radiotherapy plus temozolomide, the addition of temozolomide had no significant negative effect on QoL measures, except on social functioning ( $p=0.0052$ ) [37]. Similarly, among first-relapse GBM patients, temozolomide had no significant negative effect on QoL, although responders to temozolomide had improvement in most QoL domain scores, e.g., global, motor dysfunction, emotional function, drowsiness, future uncertainty, and communication deficit, until eventual disease progression [38-40]. These overall findings with temozolomide in GBM are concordant with the recent Cochrane review that evaluated randomized controlled trial data assessing temozolomide in patients with high-grade glioma [41].

When used as a comparator for temozolomide among the aforementioned trials, procarbazine was reported to have a negative impact on HR-QoL domain scores, e.g., drowsiness, communications deficit, motor dysfunction, role functioning, social functioning, and physical functioning, regardless of disease progression status [38, 39]. The impact of combined procarbazine, CCNU (lomustine), and vincristine (PCV) chemotherapy after radiotherapy compared with radiotherapy alone on HR-QoL measures was determined in the EORTC 26951 trial of patients with anaplastic oligodendroglioma $(n=368)$ : a major negative impact on HR-QoL (nausea/vomiting, appetite loss, and drowsiness) was found during and shortly after PCV treatment [42]. However, when HR-QoL measures were used to assess the impact of PCV chemotherapy after radiotherapy compared with radiotherapy alone in the Radiation Therapy Oncology Group (RTOG) trial 94-02 of patients with mixed anaplastic oligodendroglioma, scores were similar longitudinally and between treatments for survivors [43]. HR-QoL (Spitzer Quality of Life Index) score showed continual deterioration when measured longitudinally in the RTOB 98-03 study of escalating doses of conformal three-dimensional radiation and carmustine in GBM patients [44]. Supplementation of surgery and radiotherapy with CCNU chemotherapy provided no benefit in terms of quality of life or change in clinical performance [45]. Finally, there are very limited QoL data from randomized controlled trials with implanted carmustine-impregnated wafers in primary or recurrent high-grade glioma according to a recent Cochrane review [46].

New and investigational GBM therapies

Bevacizumab (Avastin ${ }^{\circledR}$ ), a humanized monoclonal antibody that binds to and inhibits the activity of VEGF, is the first approved antiangiogenic cancer treatment. Bevacizumab acts synergistically with cytotoxic chemotherapy or biological agents in the treatment of various tumors, e.g., colorectal, lung, renal, and breast cancer [47-50]. Recently, studies in patients with recurrent high-grade glioma or GBM have indicated promising results with durable response using the combination of bevacizumab and irinotecan [51-67], and additionally in combination with etoposide [68], nitrosourea [69], fotemustine [70], or erlotinib [71]. Positive results have also been reported with single-agent bevacizumab in recurrent high-grade glioma [57, 61, 72-74], and in combination with adjuvant temozolomide in newly diagnosed GBM [75-79].

During these studies of bevacizumab-based therapy in high-grade glioma, it was reported that one of the consequences of bevacizumab therapy is a steroid-sparing effect in a proportion of patients, which would be expected to positively impact QoL. Various studies have indicated a reduction or elimination of corticosteroid use with bevacizumab-based therapy in patients with recurrent highgrade glioma after prior treatment [53, 56-61, 63, 64, 73]. For example, in the randomized phase II clinical trial comparing bevacizumab alone or in combination with irinotecan in 167 patients with recurrent, treatment-refractory GBM [61], patients who were taking corticosteroids at baseline showed a trend to take stable or decreasing doses over time, e.g., median corticosteroid dose was reduced by about $75 \%$ after 6 months (Fig. 1). The steroid-sparing effect associated with bevacizumab-based therapy appeared associated with clinical response in high-grade glioma [58, 64] and clinical benefit such as improved neurological symptoms in high-grade glioma [59] or recurrent GBM [73].

A potentially positive impact of bevacizumab-based therapy on neurocognitive function, performance status and/or QoL has also started to emerge from reports of clinical studies among GBM patients [52, 59, 63, 64]. For example, in a retrospective analysis of recurrent GBM patients treated with bevacizumab $(n=44)$ or without bevacizumab $(n=79)$ at a single US institution [63], it was reported that bevacizumab-treated patients took longer to increase dexamethasone dose (median 149 vs. 130 days, $p=0.04)$ and also maintained their Karnofsky Performance Status (KPS) for longer (median 252 vs. 120 days, $p=0.006$ ); subgroup analysis indicated that the difference 


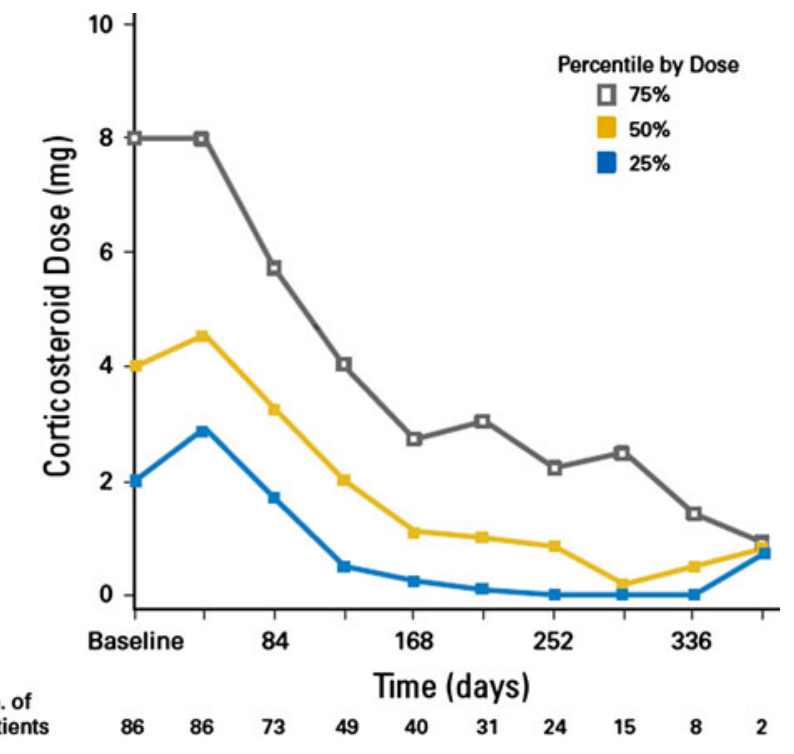

Fig. 1 Change in median corticosteroid use following treatment with bevacizumab with or without irinotecan in patients with recurrent glioblastoma multiforme (adapted with permission from Friedman et al. [61])

in these effects were more pronounced in patients aged $>55$ years. In another study of 22 consecutive patients with recurrent GBM treated with bevacizumab plus irinotecan [52], cognitive function was assessed by the Blessed Orientation-Memory-Concentration Test (BOMC) and functional status was assessed by KPS, Barthel Index (BI), and Instrumental Activities of Daily Living (IADL) prior to each cycle of treatment. Improvement in BOMC score was seen in 15 patients (62\%), with median improvement of 7 points. Improvement in functional status was seen in 18 patients $(85.7 \%)$, with median improvement in KPS by 10 points, BI by 8 points, and IADL by 2 points. The overall clinical response rate with bevacizumab plus irinotecan was $95 \%$ and was associated with significant improvements in cognitive functional and functional status.

Larger scale controlled clinical trials of bevacizumabbased therapy in GBM patients were consequently undertaken or are planned/on-going. Among these is the recently reported BRAIN study (AVF3708g), an open-label, multicenter, randomized, phase II trial of two concurrent arms treated with single-agent bevacizumab $(n=85)$ or bevacizumab plus irinotecan $(n=82)$ in patients with first- or second-relapse GBM who had been previously treated with temozolomide initially or at relapse. Primary endpoint results have been reported: estimated 6-month PFS rates were 43 and $50 \%$ in single-agent bevacizumab and bevacizumab plus irinotecan arms, respectively, and objective response rates were 28 and $38 \%$, respectively [61]. These results supported the activity of bevacizumab in recurrent GBM patients given that the 6-month PFS rate was considerably higher than the $15 \%$ rate expected for salvage chemotherapy and/or chemotherapy alone. The authors noted a trend for patients who were taking corticosteroids at baseline to take stable or decreasing doses over time, but they made no formal comparison.

More compellingly data on corticosteroid use during this study have recently been reported [80]. At baseline, 51 and $53 \%$ of patients received systemic corticosteroids in the single-agent bevacizumab and bevacizumab plus irinotecan arms, respectively. More than 75 and $65 \%$ of patients in the single-agent and combination arms, respectively, who did not receive corticosteroids at baseline continued to receive no corticosteroids after baseline. Sustained reduction in corticosteroid use was defined in this study as a $\geq 50 \%$ dose reduction for $\geq 50 \%$ of time on study treatment up to 52 weeks. Among patients with complete or partial response in the single-agent bevacizumab and bevacizumab plus irinotecan arms, 57 and 65\%, respectively, had a sustained reduction in corticosteroid use compared with 17 and 38\%, respectively, among those with stable or progressive disease.

Neurocognitive function of patients treated with singleagent bevacizumab in the BRAIN study $(n=85)$ has been analyzed [81]. Patients were assessed with the Hopkins Verbal Learning Test-Revised (HVLT-R), Trail Making Test parts A (TMTA) and B (TMTB), and the Controlled Oral Word Association (COWA) test. Assessments were conducted at baseline and then every 6 weeks while patients remained on study treatment up to 52 weeks. Change in neurocognitive function from baseline to week 6 was categorized as improved, stable, or declined, using the reliable change index. Between 93 and $98 \%$ of patients completed each test at baseline and 73-78\% completed each test at both baseline and week 6 . The majority of patients demonstrated stable performance on each test at week 6 , relative to baseline. With the exception of the COWA test, $16-28 \%$ of patients demonstrated improved performance on one or more tests at week 6 (Table 1). Preliminary results suggest that the majority of patients with recurrent GBM who were treated with bevacizumab alone in the BRAIN study demonstrated stable or improved neurocognitive function during the first 6 weeks of treatment (81-100\% across all tests). Changes across tasks and associations with measures of clinical efficacy were also explored (Table 2).

In a recent retrospective analysis [82], 12 patients with GBM and poor neurologic function (KPS $<60 \%$ ) due to bulky disease were treated in an up-front setting with bevacizumab following induction temozolomide in an attempt to improve their ability to tolerate chemoradiation. Median KPS improved from 50 to $70 \%$ and their median dexamethasone dose was reduced from 12 to $4 \mathrm{mg} / \mathrm{day}$. Five of 11 evaluable patients (45\%) had a partial response, 
Table 1 Neurocognitive changes in patients with recurrent glioblastoma receiving single-agent bevacizumab in the BRAIN study

\begin{tabular}{llll}
\hline Assessment & \multicolumn{2}{l}{$\begin{array}{l}\text { Change in performance at week 6 } \\
\text { relative to baseline (per Reliable } \\
\text { Change Index) }\end{array}$} \\
\cline { 2 - 4 } & $\begin{array}{l}\text { Improved } \\
(\%)\end{array}$ & $\begin{array}{l}\text { Declined } \\
(\%)\end{array}$ & $\begin{array}{l}\text { Stable } \\
(\%)\end{array}$ \\
\hline HVLT-R total recall $(n=74)$ & 16 & 18 & 66 \\
HVLT-R delayed recall & 14 & 16 & 70 \\
$(n=70)$ & & 19 & 59 \\
HVLT-R delayed recognition & 22 & 11 & 66 \\
$(n=69)$ & 23 & 11 & 62 \\
TMTA $(n=73)$ & 28 & 0 & 97 \\
TMTB $(n=65)$ & 3 & & \\
COWA $(n=70)$ & & 19 & \\
\hline
\end{tabular}

HVLT-R Hopkins Verbal Learning Test-Revised, TMTA Trail Making Tests part A, TMTB Trail Making Test part B, COWA Controlled Oral Word Association test

Table 2 Stabilization or improvement in neurocognitive function in patients with recurrent glioblastoma receiving single-agent bevacizumab in the BRAIN study

\begin{tabular}{lll}
\hline & $\begin{array}{l}\text { Stable/improved } \\
\text { neurocognitive } \\
\text { function on all tests } \\
n(\%)\end{array}$ & $\begin{array}{l}\text { Deterioration in } \\
\text { neurocognitive function } \\
\text { in at least one test } n(\%)\end{array}$ \\
\hline $\begin{array}{l}\text { Responders at } \\
\text { time of IRF } \\
\text { response } \\
(n=24)\end{array}$ & $18(75 \%)$ & $6(25 \%)$ \\
$\begin{array}{l}\text { PFS }>6 \text { months } \\
\text { at week } 24 \\
(n=27)^{\mathrm{a}}\end{array}$ & $19(70 \%)$ & $8(30 \%)$ \\
$\begin{array}{l}\text { Patients at time } \\
\text { of investigator }\end{array}$ & $15(31 \%)$ & $34(69 \%)$ \\
PD $(n=49)^{\mathrm{b}}$ & & \\
\hline
\end{tabular}

$I R F$ independent radiology facility, PFS progression-free survival, $P D$ progressive disease

a Two patients had missing neurocognitive data and were dropped from the analysis

b Eight patients had missing neurocognitive data and were dropped from the analysis

$4(35 \%)$ a minor response, and $1(10 \%)$ stable disease. Median TTP and OS were 5.2 and 8 months, respectively. The tumor response, rapidly improved neurologic function, and reduced steroid requirement allowed the majority of patients $(75 \%)$ to complete definitive radiotherapy.

Little significant information appears to have been published on the effects of other investigational biological agents (e.g., cilengitide, cediranib, sorafenib, sunitinib) on corticosteroid use, neurocognitive function, or QoL in patients with high-grade glioma or more specifically those with GBM. In a recent phase II study of cediranib [83], an oral pan-VEGFR tyrosine kinase inhibitor, was administered as monotherapy $(45 \mathrm{mg} /$ day) in 31 patients with recurrent GBM and resulted in encouraging proportions of radiographic partial responses of 57 and $27 \%$ on 3- and 2-dimensional MRI, respectively) and 6-month PFS (26\%). Furthermore, among 15 patients receiving corticosteroids on study entry, the dose was reduced $(n=10)$ or discontinued $(n=5)$.

\section{Conclusions}

Maintenance of QoL in patients with high-grade glioma is an important endpoint during treatment, and more so for GBM because of the particularly poor prognosis with short life expectancy at this stage of the disease. However, reliable serial measurement of QoL in patients with highgrade glioma is notoriously difficult, relating to many factors but particularly dropout bias or inability to repeatedly complete complex forms. It would appear that there is a progressive decrease in QoL during the course of highgrade glioma that substantially accelerates once the disease relapses. This is also expressed as deterioration peaks driven by the administered therapies (e.g., radiotherapy) or by the exacerbation of accompanying syndromes (e.g., brain edema, neurological symptoms, psychiatric disturbances).

It has proven difficult to identify new therapies that extend OS and PFS in patients with recurrent GBM after failure of previous therapy. Most alternative cytotoxic chemotherapeutic agents do not seem to significantly impact response or survival, yet may produce adverse effects that have a likely negative impact on QoL. However, among the new biological agents, bevacizumab has been shown to induce a clinically meaningful proportion of durable responses among patients with an acceptable safety profile. Furthermore, data are emerging that bevacizumab induces improvement or preservation of neurocognitive function, suggestive of QoL improvement, in the majority of poor-prognosis patients who would otherwise be expected to show a sudden, rapid deterioration in QoL. Further studies are underway to confirm these findings and better understand the natural history of the QoL of these patients.

Acknowledgments Support for third-party writing assistance for this manuscript, furnished by Miller Medical Communications, was provided by F. Hoffmann-La Roche Ltd.

Conflict of interest HSP has acted on an advisory board for F. Hoffmann-La Roche; RH has acted on advisory boards for F. Hoffmann-La Roche, AstraZeneca, and Schering Plough; HSP and RH have received honoraria and/or consultancy/advisory fees from F. Hoffmann-La Roche. 
Open Access This article is distributed under the terms of the Creative Commons Attribution Noncommercial License which permits any noncommercial use, distribution, and reproduction in any medium, provided the original author(s) and source are credited.

\section{References}

1. Louis DN, Ohgaki H, Wiestler OD, Cavanee WK (2007) WHO classication of tumours of the central nervous system. IARC, Lyon

2. Louis DN, Ohgaki H, Wiestler OD et al (2007) The 2007 WHO classification of tumours of the central nervous system. Acta Neuropathol 114:97-109

3. Garside R, Pitt M, Anderson R et al (2007) The effectiveness and costeffectiveness of carmustine implants and temozolomide for the treatment of newly diagnosed high-grade glioma: a systematic review and economic evaluation. Health Technol Assess 11:iii-iv, ix-221

4. Gurney JG, Kadan-Lottick N (2001) Brain and other central nervous system tumors: rates, trends, and epidemiology. Curr Opin Oncol 13:160-166

5. Stupp R, Mason WP, van den Bent MJ et al (2005) Radiotherapy plus concomitant and adjuvant temozolomide for glioblastoma. N Engl J Med 352:987-996

6. Stupp R, Hegi ME, Mason WP et al (2009) Effects of radiotherapy with concomitant and adjuvant temozolomide versus radiotherapy alone on survival in glioblastoma in a randomised phase III study: 5-year analysis of the EORTC-NCIC trial. Lancet Oncol 10:459-466

7. Wong ET, Hess KR, Gleason MJ et al (1999) Outcomes and prognostic factors in recurrent glioma patients enrolled onto phase II clinical trials. J Clin Oncol 17:2572-2578

8. Ballman KV, Buckner JC, Brown PD et al (2007) The relationship between six-month progression-free survival and 12-month overall survival end points for phase II trials in patients with glioblastoma multiforme. Neuro Oncol 9:29-38

9. Fine HA, Puduvalli VK, Chamberlain MC et al (2008) Enzastaurin (ENZ) versus lomustine (CCNU) in the treatment of recurrent, intracranial glioblastoma multiforme (GBM): a phase III study. J Clin Oncol 26(suppl; abstr 2005)

10. Lamborn KR, Yung WK, Chang SM et al (2008) Progression-free survival: an important end point in evaluating therapy for recurrent high-grade gliomas. Neuro Oncol 10:162-170

11. Macdonald DR, Kiebert G, Prados M, Yung A, Olson J (2005) Benefit of temozolomide compared to procarbazine in treatment of glioblastoma multiforme at first relapse: effect on neurological functioning, performance status, and health related quality of life. Cancer Invest 23:138-144

12. Meyers C, Boake C (1993) Neurobehavioral disorders experienced by brain tumor patients: rehabilitation strategies. Cancer Bull 45:362-364

13. Meyers CA (1986) Neuropyschologic deficits in brain-tumour patients: effects of location, chronicity, and treatment. Cancer Bull 38:20-32

14. Meyers CA, Scheibel RS (1990) Early detection and diagnosis of neurobehavioral disorders associated with cancer and its treatment. Oncology (Williston Park) 4:115-122

15. Taphoorn MJ, Klein M (2004) Cognitive deficits in adult patients with brain tumours. Lancet Neurol 3:159-168

16. Bosma I, Vos MJ, Heimans JJ et al (2007) The course of neurocognitive functioning in high-grade glioma patients. Neuro Oncol 9:53-62

17. Cheng JX, Zhang X, Liu BL (2009) Health-related quality of life in patients with high-grade glioma. Neuro Oncol 11:41-50
18. Giovagnoli AR, Silvani A, Colombo E, Boiardi A (2005) Facets and determinants of quality of life in patients with recurrent high grade glioma. J Neurol Neurosurg Psychiatry 76:562-568

19. Khuntia D, Brown P, Li J, Mehta MP (2006) Whole-brain radiotherapy in the management of brain metastasis. J Clin Oncol 24:1295-1304

20. Li J, Bentzen SM, Li J et al (2008) Relationship between neurocognitive function and quality of life after whole-brain radiotherapy in patients with brain metastasis. Int J Radiat Oncol Biol Phys 71:64-70

21. Heimans JJ, Martin J, Taphoorn B (2002) Impact of brain tumour treatment on quality of life. J Neurol 249:955-960

22. Fujii DE, Wylie AM (2003) Neurocognition and community outcome in schizophrenia: long-term predictive validity. Schizophr Res 59:219-223

23. Stong C (2003) Can neurocognition predict future quality of life in patients with mental illness? http://www.neuropsychiatryreviews. com/mar03/mar03_neurocognition.html. Accessed 5 May 2009

24. Fujii DE, Wylie AM, Nathan JH (2004) Neurocognition and long-term prediction of quality of life in outpatients with severe and persistent mental illness. Schizophr Res 69:67-73

25. Roa W, Brasher Pm, Bauman G et al (2004) Abbreviation course of radiation therap in lder patients with glioblastoma multiforme: a prospective randomized clinical trial. J Clin Oncol 22: $1582-1588$

26. Walker M, Brown J, Brown K et al (2003) Practical problems with the collection and interpretation of serial quality of life assessments in patients with malignant glioma. J Neurooncol 63:179-186

27. Correa DD (2010) Neurocognitive function in brain tumors. Curr Neurol Neurosci Rep 10:232-239

28. Klein M, Heimans JJ, Aaronson NK et al (2002) Effect of radiotherapy and other treatment-related factors on mid-term to long-term cognitive sequelae in low-grade gliomas: a comparative study. Lancet 360:1361-1388

29. Klein M, Engelberts NH, van der Ploeg HM et al (2003) Epilepsy in low-grade gliomas: the impact on cognitive function and quality of life. Ann Neurol 54:514-520

30. Taphoorn MJ (2003) Neurocognitive sequelae in the treatment of low-grade gliomas. Semin Oncol 30(6 Suppl 19):45-48

31. Klein M, Taphoorn MJ, Heimans JJ et al (2001) Neurobehavioral status and health-related quality of life in newly diagnosed highgrade glioma patients. J Clin Oncol 19:4037-4047

32. Lupien SJ, Gillin CJ, Hauger RL (1999) Working memory is more sensitive than declarative memory to the acute effects of corticosteroids: a dose-response study in humans. Behav Neurosci 113:420-430

33. Young AH, Sahakian BJ, Robbins TW, Cowen PJ (1999) The effects of chronic administration of hydrocortisone on cognitive function in normal male volunteers. Psychopharmacology (Berl) 145:260-266

34. de Vries MA, van Litsenburg RR, Huisman J et al (2008) Effect of dexamethasone on quality of life in children with acute lymphoblastic leukaemia: a prospective observational study. Health Qual Life Outcomes 6:103

35. Hempen C, Weiss E, Hess CF (2002) Dexamethasone treatment in patients with brain metastases and primary brain tumors: do the benefits outweigh the side-effects? Support Care Cancer 10: 322-328

36. Sturdza A, Millar BA, Bana N et al (2008) The use and toxicity of steroids in the management of patients with brain metastases. Support Care Cancer 16:1041-1048

37. Taphoorn MJ, Stupp R, Coens C et al (2005) Health-related quality of life in patients with glioblastoma: a randomised controlled trial. Lancet Oncol 6:937-944

38. Osoba D, Brada M, Yung WK, Prados M (2000) Health-related quality of life in patients treated with temozolomide versus 
procarbazine for recurrent glioblastoma multiforme. J Clin Oncol 18:1481-1491

39. Yung WK, Albright RE, Olson J et al (2000) A phase II study of temozolomide vs. procarbazine in patients with glioblastoma multiforme at first relapse. Br J Cancer 83:588-593

40. Brada M, Hoang-Xuan K, Rampling R et al (2001) Multicenter phase II trial of temozolomide in patients with glioblastoma multiforme at first relapse. Ann Oncol 12:259-266

41. Hart MG, Grant R, Garside R et al (2008) Temozolomide for high grade glioma. Cochrane Database Syst Rev 4:CD007415

42. Taphoorn MJ, van den Bent MJ, Mauer ME et al (2007) Healthrelated quality of life in patients treated for anaplastic oligodendroglioma with adjuvant chemotherapy: results of a European Organisation for Research and Treatment of Cancer randomized clinical trial. J Clin Oncol 25:5723-5730

43. Wang M, Cairncross G, Shaw E et al (2009) Cognition and quality of life after chemotherapy plus radiotherapy (RT) vs. RT for pure and mixed anaplastic oligodendrogliomas: radiation therapy oncology group trial 9402. J Clin Oncol 27(suppl; abstr e20519)

44. Corn BW, Wang M, Fox S et al (2009) Health related quality of life and cognitive status in patients with glioblastoma multiforme receiving escalating doses of conformal three dimensional radiation on RTOG 98-03. J Neurooncol 95:247-257

45. Trojanowski T, Peszyński J, Turowski K et al (1989) Quality of survival of patients with brain gliomas treated with postoperative CCNU and radiation therapy. J Neurosurg 70:18-23

46. Hart MG, Grant R, Garside R et al (2008) Chemotherapeutic wafers for high grade glioma. Cochrane Database Syst Rev 3:CD007294

47. Yang JC, Haworth L, Sherry RM et al (2003) A randomized trial of bevacizumab, an anti-vascular endothelial growth factor antibody, for metastatic renal cancer. N Engl J Med 349:427-434

48. Hurwitz H, Fehrenbacher L, Novotny W et al (2004) Bevacizumab plus irinotecan, fluorouracil, and leucovorin for metastatic colorectal cancer. N Engl J Med 350:2335-2342

49. Sandler A, Gray R, Perry MC et al (2006) Paclitaxel-carboplatin alone or with bevacizumab for non-small-cell lung cancer. N Engl J Med 355:2542-2550

50. Miller K, Wang M, Gralow J et al (2007) Paclitaxel plus bevacizumab versus paclitaxel alone for metastatic breast cancer. N Engl J Med 357:2666-2676

51. Stark-Vance V (2005) Bevacizumab and CPT-11 in the treatment of relapsed malignant glioma. Neuro Oncol 7:369 (abstr 342)

52. Raval S, Hwang S, Dorsett L (2007) Bevacizumab and irinotecan in patients (pts) with recurrent glioblastoma multiforme (GBM). J Clin Oncol 25(suppl; abstr 2078)

53. Vredenburgh JJ, Desjardins A, Herndon JE 2nd et al (2007) Bevacizumab plus irinotecan in recurrent glioblastoma multiforme. J Clin Oncol 25:4722-4729

54. Vredenburgh JJ, Desjardins A, Herndon JE 2nd et al (2007) Phase II trial of bevacizumab and irinotecan in recurrent malignant glioma. Clin Cancer Res 13:1253-1259

55. Ali SA, McHayleh WM, Ahmad A et al (2008) Bevacizumab and irinotecan therapy in glioblastoma multiforme: a series of 13 cases. J Neurosurg 109:268-272

56. Bokstein F, Shpigel S, Blumenthal DT (2008) Treatment with bevacizumab and irinotecan for recurrent high-grade glial tumors. Cancer 112:2267-2273

57. Cloughesy TF, Prados MD, Wen PY et al (2008) A phase II, randomized, non-comparative clinical trial of the effect of bevacizumab (BV) alone or in combination with irinotecan (CPT) on 6-month progression free survival (PFS6) in recurrent, treatmentrefractory glioblastoma (GBM). J Clin Oncol 26(suppl; abstr 2010b)

58. Desjardins A, Reardon DA, Herndon JE 2nd et al (2008) Bevacizumab plus irinotecan in recurrent WHO grade 3 malignant gliomas. Clin Cancer Res 14:7068-7073
59. Guiu S, Taillibert S, Chinot O et al (2008) Bevacizumab/irinotecan. An active treatment for recurrent high grade gliomas: preliminary results of an ANOCEF Multicenter Study. Rev Neurol (Paris) 164:588-594

60. Norden AD, Young GS, Satayesh K et al (2008) Bevacizumab for recurrent malignant gliomas: efficacy, toxicity, and patterns of recurrence. Neurology 70:779-787

61. Friedman HS, Prados MD, Wen PY et al (2009) Bevacizumab alone and in combination with irinotecan in recurrent glioblastoma. J Clin Oncol 27:4733-4740

62. Gilbert MR, Wang M, Aldape K et al (2009) RTOG 0625: A phase II study of bevacizumab with irinotecan in recurrent glioblastoma (GBM). J Clin Oncol 27(suppl; abstr 2011)

63. Nghiemphu PL, Liu W, Lee Y et al (2009) Bevacizumab and chemotherapy for recurrent glioblastoma: a single-institution experience. Neurology 72:1217-1222

64. Poulsen HS, Grunnet K, Sorensen M et al (2009) Bevacizumab plus irinotecan in the treatment patients with progressive recurrent malignant brain tumours. Acta Oncol 48:52-58

65. Zuniga RM, Torcuator R, Jain R et al (2009) Efficacy, safety and patterns of response and recurrence in patients with recurrent high-grade gliomas treated with bevacizumab plus irinotecan. J Neurooncol 91:329-336

66. Cloughesy T, Vredenburgh JJ, Day B et al (2010) Updated safety and survival of patients with relapsed glioblastoma treated with bevacizumab in the BRAIN study. J Clin Oncol 28(suppl; abstr 2008)

67. Desjardins A, Vredenburgh JJ, Reardon DA et al (2010) Longterm survival from the initial trial of bevacizumab and irinotecan. J Clin Oncol 28(suppl; abstr 2045)

68. Reardon D, Desjardins A, Vredenburgh JJ et al (2009) Bevacizumab plus etoposide among recurrent malignant glioma patients: phase II study final results. J Clin Oncol 27(suppl; abstr 2046)

69. Soffietti R, Rudà R, Trevisan E et al (2009) Phase II study of bevacizumab and nitrosourea in patients with recurrent malignant glioma: A multicenter Italian study. J Clin Oncol 27(suppl; abstr 2012)

70. Trevisan E, Picco E, Rudà R et al (2009) Salvage therapy with bevacizumab and fotemustine in recurrent high grade glioma. Eur J Can Suppl 7:499(abstr P-8719)

71. Sathornsumetee S, Desjardins A, Vredenburgh JJ et al (2010) Phase II study of bevacizumab plus erlotinib for patients with recurrent malignant gliomas: final results. J Clin Oncol 28(suppl; abstr 2055)

72. Gutin PH, Iwamoto FM, Beal K et al (2009) Safety and efficacy of bevacizumab with hypofractionated stereotactic irradiation for recurrent malignant gliomas. Int $\mathrm{J}$ Radiat Oncol Biol Phys 13:1200-1206

73. Kreisl TN, Kim L, Moore K et al (2009) Phase II trial of singleagent bevacizumab followed by bevacizumab plus irinotecan at tumor progression in recurrent glioblastoma. J Clin Oncol 27:740-745

74. Raizer JJ, Grimm S, Rice L et al (2009) A phase II trial of singleagent bevacizumab given every 3 weeks for recurrent malignant gliomas. J Clin Oncol 27(suppl; abstr 2044)

75. Lai A, Filka E, McGibbon B et al (2008) Phase II pilot study of bevacizumab in combination with temozolomide and regional radiation therapy for up-front treatment of patients with newly diagnosed glioblastoma multiforme: interim analysis of safety and tolerability. Int J Radiat Oncol Biol Phys 71:1372-1380

76. Gruber ML, Raza S, Gruber D et al (2009) Bevacizumab in combination with radiotherapy plus concomitant and adjuvant temozolomide for newly diagnosed glioblastoma: update progression-free survival, overall survival, and toxicity. J Clin Oncol 27(suppl; abstr 2017) 
77. Nicholas MK, Lucas RV, Arzbaecher J et al (2009) Bevacizumab in combination with temozolomide in the adjuvant treatment of newly diagnosed glioblastoma multiforme: preliminary results of a phase II study. J Clin Oncol 27(suppl; abstr 2016)

78. Vredenburgh JJ, Desjardins A, Reardon DA et al (2010) Bevacizumab (BEV) in combination with temozolomide (TMZ) and radiation therapy (XRT) followed by BEV, TMZ, and irinotecan for newly diagnosed glioblastoma multiforme (GBM). J Clin Oncol 28(suppl; abstr 2023)

79. Omuro AM, Beal K, Karimi S et al (2010) Phase II study of bevacizumab (BEV), temozolomide (TMZ), and hypofractionated stereotactic radiotherapy (HFSRT) for newly diagnosed glioblastoma (GBM). J Clin Oncol 28(suppl; abstr 2036)

80. Vredenburgh JJ, Wefel J, Coughesy T et al (2009) Clinical assessment of corticosteroid use and neurocognitive function in patients with glioblastoma at first or second relapse treated with bevacizumab in the BRAIN study. Eur J Cancer Suppl 7:495 (abstr O-8701)

81. Wefel JS, Cloughesy T, Zazzali J et al (2009) Neurocognitive function in patients with glioblastoma multiforme in first or second relapse treated with bevacizumab in the BRAIN study. J Clin Oncol 27(suppl; abstr 2056)

82. Green RM, Woyshner EA, Nghiemphu L et al (2010) Use of bevacizumab to faciliate up-front chemoradiation in poor-risk patients with glioblastoma multiforme by improving neurologic function. J Clin Oncol 28(suppl; abstr 2059)

83. Batchelor TT, Duda DG, di Tomaso E et al (2010) Phase II study of cediranib, an oral pan-vascular endothelial growth factor receptor tyrosine kinase inhibitor, in patients with recurrent glioblastoma. J Clin Oncol 28:2817-2823 